\title{
Development of a generalised equilibrium modified atmosphere model and its application to the $\mathbf{t}$ Taleggio ollos cheese
}

(i) The corrections made in this section will be reviewed and approved by a journal production editor.

Federico Florit, Andrea Fiorati, Filippo Ghisoni, Gabriele Pozzoli, Renato Rota, Luigi De Nardo*

luigi.denardo@polimi.it

Politecnico di Milano, Dipartimento di Chimica, Materiali e Ingegneria Chimica G. Natta, Piazza Leonardo da Vinci, 32, 20133, Milano, Italy

* Corresponding author.

\begin{abstract}
The food metabolic processes influence the gas composition of packaged products: by finely tuning the gas fluxes through the packaging, the aerobic and anaerobic respiration processes can be efficiently exploited to regulate the equilibrium gas concentrations. In this work, we present a generalised model able to predict the evolution of gases in micro-perforated equilibrium MAP, with a detailed evaluation of fluxes through the perforations by means of Computational Fluid Dynamics. It was found that the Sherwood number for the studied micro-perforations is 0.715 and it was confirmed via experiments on packaging with oxygendepleted atmospheres. The kinetic model was experimentally validated on a smear short-ripened soft cheese (Taleggio) whose complex surface microbiota confer to the product a non-trivial respiration behaviour. Cheese slices were packed with three different micro-perforated solutions (one $120 \mu \mathrm{m}$ diameter hole, two $90 \mu \mathrm{m}$ diameter holes, and five $90 \mu \mathrm{m}$ diameter holes) achieving three different equilibrium gas compositions with good model predictions. The model was applied to literature data with success, thus the model can be deemed general and applicable to many different systems.
\end{abstract}

\section{Keywords:}

Equilibrium modified atmosphere packaging, Micro-perforation, Food packaging, Mathematical modelling

\section{Nomenclature}

\section{Symbols}

$\mathscr{A}$ Area

$C_{D} \quad$ Discharge coefficient

$C W L \quad$ Percent cumulative weight loss

D Diffusivity

$d$ Diameter

$J \quad$ Material flux

$K_{m, O_{2}} \quad$ Michaelis-Menten constant for $\mathrm{O}_{2}$

$\mathrm{K}_{\mu, \mathrm{O}_{2}}$ Inhibition constant for $\mathrm{O}_{2}$

$K_{1}, K_{2}$ Inhibition constants for $\mathrm{CO}_{2}$

$l$ Membrane thickness

$M W \quad$ Molecular weight

$N_{H} \quad$ Number of micro-perforations

$N C$ Number of components (species)

$P \quad$ Pressure

$P^{0} \quad$ Vapour pressure 
$R \quad$ Ideal gas constant

$R_{i} \quad$ Production rate for species $i$

$R Q \quad$ Respiratory quotient

Sh Sherwood number

$T$ Temperature

$t$ Time

$V$ Volume

$v_{O_{2}, \max }$ Maximum $\mathrm{O}_{2}$ respiration rate

$v_{\mathrm{CO}_{2}, \mathrm{f}}$ Maximum anaerobic respiration rate

$W$ Food weight

WVT Water vapour transmission

$x$ Axial coordinate

$y$ Molar fraction

\title{
Greek symbols
}

$\alpha$ Water production rate constant

$\Delta h^{e v} \quad$ Evaporation enthalpy

$\rho^{L} \quad$ Liquid density

\section{Subscripts and superscripts}

\author{
$a d v$ Advection \\ atm Atmospheric \\ cond Condensed \\ $b$ Bulk \\ C Condensation \\ $H$ Hole/perforation \\ i Species \\ M Membrane
}

\section{Introduction}

Food packaging is pervasive and essential in modern food industry: it contains and protects food and beverages, from manufacturing to the final consumer (Ruggeri et al., 2020). The extension of the shelf-life through the optimisation of the packaging based on the product requirements has become a powerful strategy for both supporting marketing needs and reducing food waste or loss (Ciccullo et al., 2021; Bossi et al., 2016). Modified Atmosphere Packaging (MAP), especially when combined with low temperature storage, has been effectively proven to modulate the metabolic rate of different food produeeproducts, by slowing their spoilage kinetic (Zeuthen et al., 2002). MAP was proven to be useful in prolonging the shelf life of dairy products in terms of microbiological and sensorial aspects (Marcos et al., 2016; Rodriguez-Aguilera and Oliveira, 2009; Esmer et al., 2009; Papaioannou et al., 2007; Dermiki et al., 2008). The dairy industry represents one of the major food sectors in Italy, cheese being one of the most important dairy products, both in terms of quantity and quality (Zeuthen et al., 2002). This industrial sector is taking advantage of the MAP approach. Cheese is a complex system where different reactions take place during both its manufacturing and storage, being susceptible to physical, chemical, and biochemical deterioration (Son, 2013 Robertson, 2012). Cheese constituents undergo several changes by the action of the residual enzymes as well as the activity and the growth of starter and nonstarter microorganisms (Youssef et al., 2017). If not correctly managed, biochemical and microbiological processes that influences the cheese quality will lead to variable and detrimental defects in the final product. In order to design a proper packaging system for cheeses, it is mandatory to provide an atmosphere that is favourable to the involved microorganisms to avoid the development of off-flavors, the control of both $\mathrm{CO}_{2}$ and $\mathrm{O}_{2}$ concentration being therefore crucial (Rodriguez-Aguilera and Oliveira, 2009). This is even more important for mould ripened cheeses. The initial presence of mould adds complexity to this kind of systems: the gas dynamics inside the package will be determined by both aerobic respiration and anaerobic respiration (fermentation). Taleggio is a smear short-ripened soft cheese with washed rind produced in the Taleggio valley of Lombardy (Italy), granted Protected Denomination of Origin (PDO). It is characterised by complex surface microbiota, which contribute to its typical sensorial characteristics (Fontana et al., 2010; Gobbetti et al., 1997; Firloni et al., GhelardiTirloni et al.,_2020).

Optimal gas concentration and humidity range have been reported to optimise the shelf life in MAP products (Son, 2013Robertson, 2012; Khoshgozaran et al., 2012). In particular, Equilibrium Modified Atmosphere Packaging (EMAP), based on modulating the in-package equilibrium atmosphere by a suitable design of the mass transport processes, represents a simple yet effective strategy to obtain self-regulated head space gas composition, achieving a significant extension of the primary shelf-life (Hussein et al., 2015). However, a possible water accumulation at the 
product surface could promote microbial growth and sliminess, thus impairing the objective of the shelf-life extension ( Sousa-Gallagher et al., 2013). Along this direction, the combination of EMAP with micro-perforations represents a valid alternative to MAP. Polymeric films commonly used for MAP applications have low values of $O_{2}$ permeability ( Marcos et al., 2016; Winotapun et al., 2015). This represents an important limitation of MAP for highly respiring products, because a combination of low oxygen permeability and a high respiration rate easily leads to anaerobic conditions inside the package. Perforations in the polymeric film represent hence the easiest and effective option to enhance the gas and vapour transport across the barrier, being micro-holes a parallel route for gas transport that increases the total apparent permeability of the material. The synergy that is created between the material's intrinsic properties and the flexibility provided by perforations somehow creates a new structure that represents an optimised version of the pristine material (Winotapun et al., 2015).

In order to design the perforations for optimal EMAP, a predictive model can be developed to describe the gas composition during storage. Several models are already available in literature, mainly focusing on the respiration of fruits and vegetables, thus aiming at predicting $\mathrm{O}_{2}$ and $\mathrm{CO}_{2}$ composition changes in time. Common assumptions of such models are the uniformity of gas composition and temperature in the system, together with constant volume of the packaging and pressure inside of it; temperature is often assumed to be constant (or given) (Geysen et al., 2007; Rodriguez-Aguilera et al., 2009; Ghosh and Dash, 2020) even if models with non-constant temperature exist (Song et al., 2002). More complex models have been developed to describe also the space gradients in composition (Rennie and Tavoularis, 2009a, 2009bbib_Rennie_and_Tavoularis_2009abib_Rennie_and_Tavoularis_2009b; Xanthopoulos et al., 2012), but require additional computational efforts. The assumption of constant volume is not always verified, such as when the packaging is not rigid (e.g., a plastic bag) and models have been developed to consider this kind of systems ( Castellanos et al., 2016a). Other models also consider water (Castellanos et al., 2016b) or ethylene (Castellanos et al., 2017) production during respiration. Most of the aforementioned models use respiration rate expressions based on the Michaelis-Menten (MM) mechanism. This kind of reaction rates is almost always suitable for the description of produce respiration under aerobic conditions or under low (non null) concentration of oxygen.

The aim of this work is to develop a generalised model able to describe both aerobic and anaerobic conditions for MAP (focusing especially on dairy products) with an arbitrary number of modelled chemical species, under isothermal conditions. The volume and total pressure of the package will be modelled as well, thus they will not be assumed constant. Fluxes through the micro-perforations will be estimated using computational fluid dynamics (CFD). The

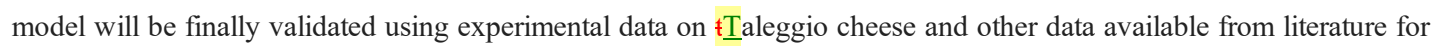
another soft cheese.

\section{Materials and methods}

\subsection{Materials}

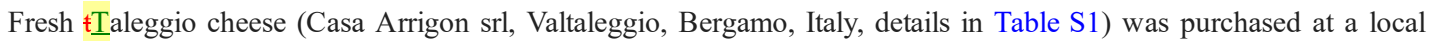
grocery supermarket prior to the start of the experiments. Taleggio cheese was packed in commercial poly (ethylene terephthalate) (PET) trays $\left(184 \mathrm{~mm}^{3} \times 117 \mathrm{~mm}^{3} \times 40 \mathrm{~mm}^{3}\right.$, thickness $\left.250 \mu \mathrm{m}\right)$, sealed by means of a PET-based film possessing a nominal thickness of $17 \mu \mathrm{m}$ and a permeable area of $215 \mathrm{~cm}^{2}$, an oxygen permeance of 150 $\mathrm{cm}^{3} \mathrm{~m}^{-2} \mathrm{~d}^{-1}$ bar $^{-1}$ and a carbon dioxide permeance of $640 \mathrm{~cm}^{3} \mathrm{~m}^{-2} \mathrm{~d}^{-1}$ bar $^{-1}$. More details about PET-Based film properties are reported in Table $\mathrm{S} 2$.

PET films were perforated by means of a laser perforation system, obtaining holes with diameters of $90 \mu \mathrm{m}$ and $120 \mu \mathrm{m}$ respectively. Micro-perforated PET lids have been labeled as PETX-Y, where X represents the number of holes ( $\mathrm{X}$ being 1 or 5 ) and $\mathrm{Y}$ the nominal hole diameter in $\mu \mathrm{m}$ (Y being 90 or 120), as reported in Table 1.

alt-text: Table 1

\section{Table 1}

(i) The table layout displayed in this section is not how it will appear in the final version. The representation below is solely purposed for providing corrections to the table. To preview the actual presentation of the table, please view the Proof.

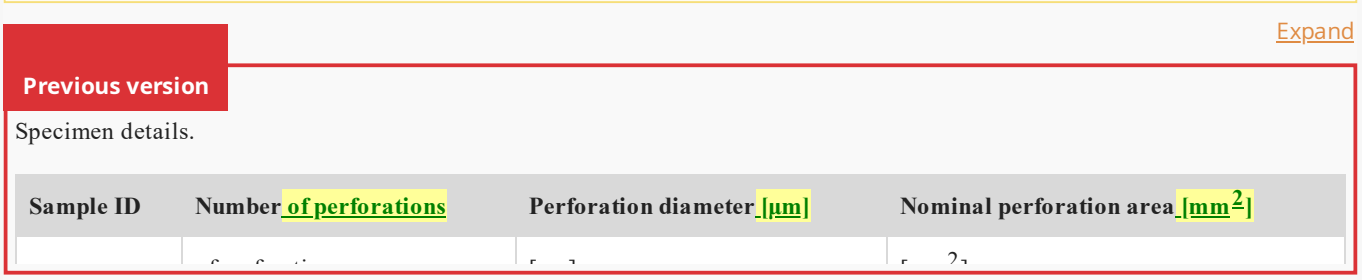




\begin{tabular}{|c|c|c|c|}
\hline Sample ID & Numberof perforations & Perforation diameter_[ $[\underline{\mu \mathrm{m}}]$ & Nominal perforation area_[ $\left.\underline{\mathrm{mm}}^{\underline{2}}\right]$ \\
\hline PET & 0 & & \\
\hline PET1-120 & 1 & 120 & $1.13 \times 10^{-2}$ \\
\hline PET2-90 & 2 & 90 & $1.27 \times 10^{-2}$ \\
\hline PET5-90 & 5 & 90 & $3.18 \times 10^{-2}$ \\
\hline
\end{tabular}

\subsection{Model development}

The time evolution of the gas composition inside the packaging can be described through molar fraction balance equations. A scheme of the modelled system is reported in Fig. 1. It is assumed that temperature, $T$, is uniform and constant, while the gases do not show concentration gradients inside the package (i.e., they are assumed to be perfectly mixed). The gases are assumed to behave as ideal gases.

alt-text: Fig. 1

Fig. 1

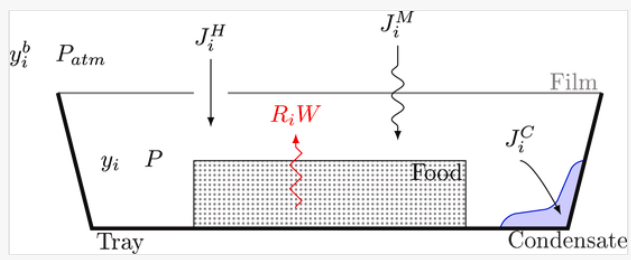

[Instruction: This figure is far from where it is first referenced to in the proof/pdf.]Scheme of modelled system reporting the composition and fluxes of species $i . H$ refers to micro-perforations and $M$ to the film/membrane.

By a species mole balance equation it is possible to write the change of composition in time, $t$ :

$$
\frac{d y_{i}}{d t}=\frac{1}{V}\left(J_{i}+R_{i} W\right)-\frac{y_{i}}{V} \frac{d V}{d t}-\frac{y_{i}}{P} \frac{d P}{d t}
$$

where $y_{i}$ is the molar fraction of species $i$ in the headspace (for $i=1 \ldots N C$, being $N C$ the number of modelled chemical compounds in the system), $V$ the headspace volume, $J_{i}$ the net flux of species $i, R_{i}$ the-(signed, pesitive for products and negative for reagents) production rate_(signed, positive for products and negative for reagents) of species $i$ per unit food mass, $W$ the mass of food inside the package, and $P$ the total pressure inside the package. It should be noted that the time variation of volume and pressure is considered. In Equation (1) the net species flux is given by the contributions of the membrane (M) permeability, possible presence of $N_{H}$ micro-perforations $(\mathrm{H})$ on the membrane, and condensation $(\mathrm{C})$ :

$$
J_{i}=J_{i}^{M}+N_{H} J_{i}^{H}-J_{i}^{C}
$$

It is assumed that the gas does not permeate through the walls of the tray. The membrane permeability contribution to the net flux can be described by:

$$
J_{i}^{M}=\frac{\mathscr{P}_{i} \mathscr{A}_{M} P}{l}\left(\frac{P_{a t m}}{P} y_{i}^{b}-y_{i}\right)
$$

where $\mathscr{D}_{i}$ is the permeability of the membrane to species $i, \mathscr{A}_{M}$ the surface of the membrane, $l$ its thickness, $P_{a t m}$ the (constant) atmospheric pressure, and $y_{i}^{b}$ the (constant bulk) molar fraction of species $i$ outside the package.

The contribution given by the perforations can be described by two terms, the first describing diffusion through the hole, the other describing advection due to a pressure difference between the package and the outer atmosphere:

$$
J_{i}^{H}=\frac{\mathscr{D}_{i} S h}{d_{H}} \frac{\pi d_{H}^{2}}{4}\left(\frac{P_{a t m}}{P} y_{i}^{b}-y_{i}\right)+J_{i}^{a d v}
$$


where $\mathscr{D}_{i}$ is the diffusivity of species $i$ (reported in the Supporting Information), Sh the Sherwood number (which will be determined in the following sections), $d_{H}$ the hole diameter, and $J_{i}^{a d v}$ the advection contribution given by:

$$
J_{i}^{a d v}= \begin{cases}-C_{D} y_{i} \frac{\pi d_{H}^{2}}{4} \sqrt{2 \frac{R T}{M W}\left(\frac{P}{P_{a t m}}-1\right)} & P>P_{a t m} \\ 0 & P=P_{a t m} \\ C_{D} y_{i}^{b} \frac{\pi d_{H}^{2}}{4} \sqrt{2 \frac{R T}{M W^{b}}\left(\frac{P_{a t m}}{P}-1\right)} & P<P_{a t m}\end{cases}
$$

[Instruction: Equation 5 seems to have a blank first line inside the bracket in the generate proof/pdf. It should be deleted.]

where $C_{D}$ is the discharge coefficient, $M W$ the molecular weight of the gas in the headspace, and $M W^{b}$ the molecular weight of the gas in the outer atmosphere. It was assumed that the advection is due to an incompressible flow (which is generally the case, as the Mach number of this kind of systems is lower than 0.3, indicating an incompressible flow ( Panton, 2013)). The discharge coefficient becomes quite low for this kind of flow and can be evaluated from correlations (Hollingshead et al., 2011). The value of $C_{D}=0.003$ will be used as it provides a good estimate for very low flowrates such as those achieved in micro-perforated MAP.

The contribution due to condensation is null for all incondensable gases (e.g., oxygen and nitrogen) and is assumed to be null for all condensable gases which are not at saturation conditions. When a condensable gas $k$ reaches saturation, $J_{k}^{C}$ is such that the molar fraction is kept equal to the saturation value over time, meaning $y_{k}=P_{k}^{0}(T) / P$ (where $P_{k}^{0}(T)$ is the vapour pressure of species $k$ at some temperature $T$ ), and by differentiation:

$$
\frac{d y_{k}}{d t}=-\frac{P_{k}^{0}(T)}{P^{2}} \frac{d P}{d t}
$$

and by comparison with Equation (1) an algebraic equation is obtained to determine the value of $J_{k}^{C}$. This equation can be simplified to obtain an explicit expression of the condensation flux when volume or pressure are kept constant, for which the details are reported in the Supporting Information.

The condensed species will form a liquid phase of changing volume in time:

$$
\frac{d V_{\text {cond }}}{d t}=\sum_{i}^{N C} \frac{J_{i}^{C} M W_{i} P}{R T \rho_{i}^{L}}
$$

where $M W_{i}$ is the molecular weight of species $i$ and $\rho_{i}^{L}$ is the density of pure species $i$ in the liquid phase. (The assumption of ideal liquid mixture was used for this last equation.) In the following, only water condensation will be considered, therefore allowing to simplify correspondingly the previous equation.

By summation of Equation (1) over all species one obtains:

$$
\frac{d V}{d t}+\frac{V}{P} \frac{d P}{d t}=\sum_{i}^{N C}\left(J_{i}+R_{i} W\right)
$$

This equation provides the description of the pressure/volume variation in time if a suitable model is used to describe the link between volume and pressure. This link can be for example a mechanical model of the membrane deforming due to changes in pressure, thus a form such as $V=V(P)$.

For this work, unless a large build-up of pressure is attained, the package can be safely assumed to be of constant volume, i.e., $d V / d t=0$. This corresponds to the assumption of a rigid package, which is the case as most of the package is built with a rigid plastic. The opposite behaviour is the one of a totally flexible membrane for which pressure can be assumed to be constant, i. e, $d P / d t=0$. This is the case for packaging made by a flexible bag, such as those used in (Castellanos et al., 2016a, 2016bbib_Castellanos_et_al_2016abib_Castellanos_et_al_2016b). Therefore, the proposed model can describe both rigid packaging and flexibe bags, as well as intermediate conditions for which a mechanical model should be provided. Therefore, the proposed model is a general description of food packaging able to describe very diverse situations. 
In the following, the headspace volume will be assumed to be constant, thus the first therm on the L.H.S. of Equation

(8) is null and the equation can be used to describe the pressure evolution in time inside the package.

As reaction products may leave the food as gases, the food mass changes in time:

$$
\frac{d W}{d t}=-\sum_{i}^{N C} R_{i} W \frac{M W_{i} P}{R T}
$$

It is common practice to observe the percent cumulative weight loss, defined as:

$$
C W L=\frac{W(t=0)-W}{W(t=0)} \cdot 100
$$

The reactions considered in the system are aerobic and anaerobic respiration. The model proposed can describe an arbitrary number of chemical species. In this work four species are considered: oxygen, carbon dioxide, water, and nitrogen (for the latter the reaction rate is null). $\mathrm{O}_{2}$ is consumed due to aerobic respiration, which produces $\mathrm{CO}_{2}$ and $\mathrm{H}_{2} \mathrm{O}$. Anaerobic respiration mainly produces $\mathrm{CO}_{2}$. Both reactions can be inhibited by the presence of $\mathrm{O}_{2}$ and $\mathrm{CO}_{2}$. In order to take this into account, respiration rates for oxygen can be described through Michaelis-Menten kinetic schemes in the uncompetitive-inhibition formulation (MMU). The aerobic respiration rate for $\mathrm{CO}_{2}$ can be obtained from the so-called respiratory quotient, describing the ratio between the respiration rate of carbon dioxide and the one of oxygen under aerobic conditions. Anaerobic respiration reactions are generally neglected in literature and are somehow taken into account in the respiratory quotient when its value is large, as typical values of $R Q$ for aerobic respiration range between 0.7 and 1.3 (Song et al., 2002). This description can be erroneous given the MMU equations, especially for dairy products. The MMU would predict a null production rate for $\mathrm{CO}_{2}$ if no oxygen is present. This is clearly not the case when anaerobic respiration becomes relevant. Therefore, a separate term in the reaction rate was used to describe it (inhibited by high concentration of both $\mathrm{O}_{2}$ and $\mathrm{CO}_{2}$ ) resembling a noncompetitive MichaelisMenten rate. The following expressions use parameters given at a single temperature. An Arrhenius' law can be used for the description of the temperature variation of such parameters. In this work, as all experiments were run at the same temperature, hence there is no need to use such temperature-dependent kinetic constants.

The production rate for $\mathrm{O}_{2}$ is described by the MMU only:

$$
R_{O_{2}}=-\frac{v_{O_{2}, \max } y_{O_{2}}}{K_{m, O_{2}}+y_{O_{2}}\left(1+\frac{y_{\mathrm{CO}_{2}}}{K_{\mu, O_{2}}}\right)}
$$

where $v_{O_{2}, \max }$ is the maximum $O_{2}$ respiration rate, $K_{m, O_{2}}$ the $\mathrm{MM}$ constant for oxygen, and $K_{\mu, O_{2}}$ the inhibition constant due to carbon dioxide.

The production rate for $\mathrm{CO}_{2}$ also has an additional term for anaerobic respiration:

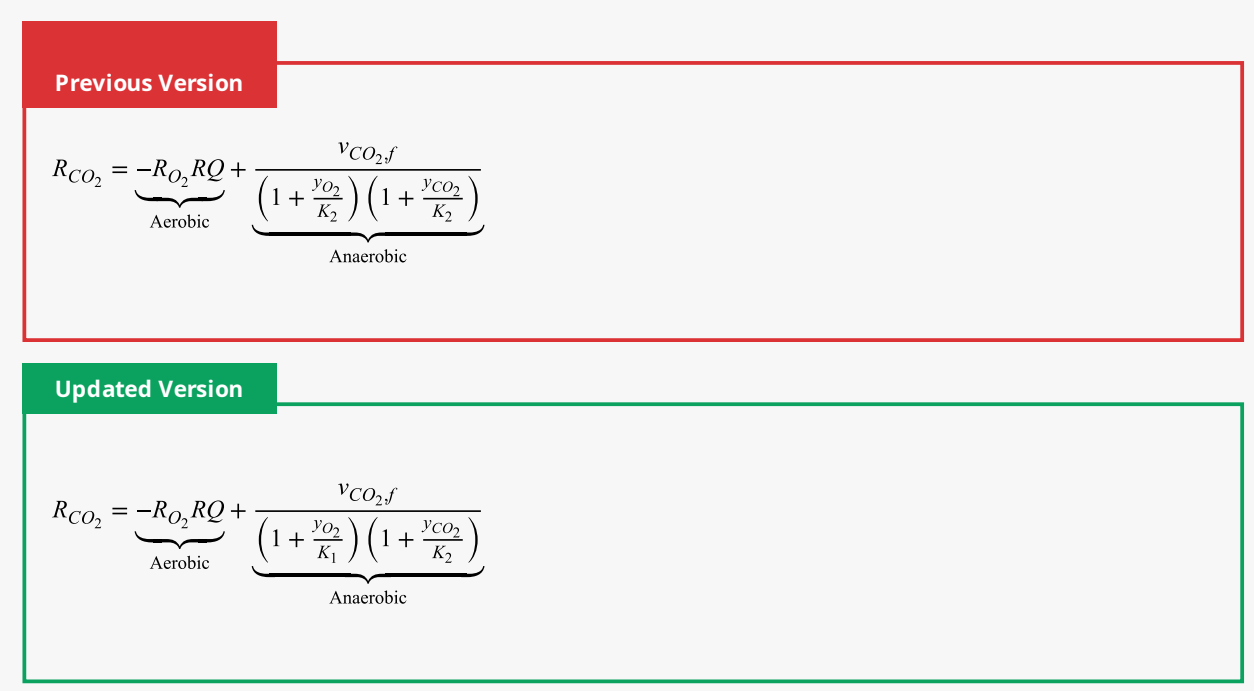


where ${ }^{C_{2}, f}$ is the maximum anaerobic respiration rate, $K_{1}$ and $K_{2}$ are parameters taking into account inhibition by $\mathrm{O}_{2}$ and $\mathrm{CO}_{2}$ respectively.

The production rate for $\mathrm{H}_{2} \mathrm{O}$ can be computed as an average of the aerobic respiration rates of oxygen and carbon dioxide multiplied by a factor taking into account the respiration reaction stoichiometry (Song et al., 2002):

$$
R_{\mathrm{H}_{2} \mathrm{O}}=-\alpha\left(\frac{2816\left[\mathrm{~kJ} \mathrm{~mol}^{-1}\right]}{6 \Delta h_{\mathrm{H}_{2} \mathrm{O}}^{e v}}\right) R_{\mathrm{O}_{2}} \frac{1+R Q}{2}
$$

where $\alpha$ is a parameter describing the fraction of respiration energy transformed into heat and $\Delta h_{\mathrm{H}_{2} \mathrm{O}}^{e v}$ the evaporation enthalpy of water. Generally, the value of $\alpha$ ranges from 0.80 to 1.00 (Song et al., 2002) but values as low as 0.25 were reported (Castellanos et al., 2016b).

\subsection{CFD modelling}

CFD simulations were performed to evaluate the purely diffusive flux across the perforations using the Ansys Fluent 19.1 software. The simulations were performed for the selected membrane (thickness $17 \mu \mathrm{m}$ ) and the two diameters considered $(90$ and $120 \mu \mathrm{m})$. A 2D domain was considered by exploiting the cylindrical symmetry of a single hole. The membrane divides space in two regions (the headspace and the outer atmosphere) for which two compositions were assigned and kept constant at a large distance from the hole. For each species the difference between the two assigned compositions, $\Delta y_{i}$, is kept constant while solving the molar fraction transport equations. The details on the computational domain and solver settings are reported in the Supporting Information.

The diffusive flux was evaluated from the spatial derivative of molar fraction at the centre of the hole:

Previous Version

$J_{i}^{H}=\frac{\pi d_{H}^{2}}{4} \mathscr{D}_{i} \frac{\partial y_{i}}{\partial x}{ }_{x=0}$

Updated Version

$J_{i}^{H}=\left.\frac{\pi d_{H}^{2}}{4} \mathscr{D}_{i} \frac{\partial y_{i}}{\partial x}\right|_{x=0}$

The pressure was imposed to be uniform in order not to have convection in the system.

\subsection{Validation experiments}

The computed $\mathrm{O}_{2}$ fluxes were validated by sealing the PET-based tray with different specimens in a low $\mathrm{O}_{2}(2-5 \%)$ environment, then the samples were stored at $5{ }^{\circ} \mathrm{C}, 40 \% \mathrm{RH}$ for 20 days. Daily oxygen measurements were made using a non-destructive approach by means of $\mathrm{OpTech}^{\circledast}-\mathrm{O}_{2}$ Platinum Oxygen Analyzer (Mocon INC, Minneapolis, MN, US).

Taleggio cheese slices (200 g), carefully sliced to have homogeneous samples in terms of creamy part and crust, was packed using PET trays and sealed with PET-based films using an industrial sealing machinery (Proseal GTOe, Proseal, Adlington, Cheshire, UK), resulting in an average headspace of $600 \mathrm{~cm}^{3}$. During the packaging, unmodified air atmosphere conditions were used. Different micro-perforated PET-based lids were employed to achieve different oxygen equilibrium concentration. Each sample was stored at $8{ }^{\circ} \mathrm{C}, 67 \% \mathrm{RH}$ for $55 \mathrm{~d}$. The internal atmosphere composition evolution was measured by means of gas analyser Dansensor CheckPoint 3 (Dansensor ${ }^{\circledR}$, Mocon INC, Minneapolis, MN, US).

\section{Results and discussion}

\subsection{Micro-perforation characterisation}

The CFD simulations provide the spatial distribution of species in the domain. Contours of the molar fraction of one species $\left(\mathrm{CO}_{2}\right)$ close to the micro-perforation are reported in Fig. 2 for the two hole diameters considered. It can be observed that the molar fraction gradients vanish quickly and can be considered to be null at a distance of 
approximately 3 diameters from the centre of the orifice. Therefore, multiple perforations separated by such distance can be considered to be independent on each other. This is the case of the experimental setup, where the perforations are separated by several centimetres of distance. Additionally, the results obtained agree qualitatively (in terms of contours shape) with those reported in (Rennie and Tavoularis, 2009b).

alt-text: Fig. 2

\section{Fig. 2}

Figure Replacement Requested
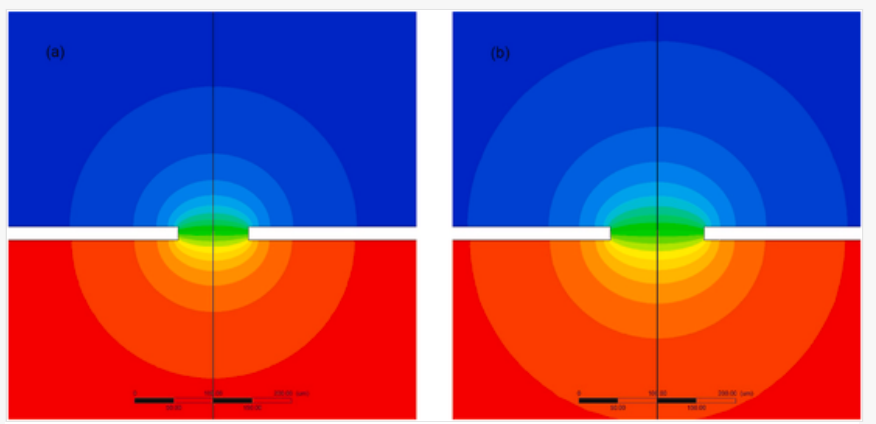

[Instruction: This figure is far from where it is first referenced to in the proof/pdf.]Mole fraction distribution of $\mathrm{CO}_{2}$ across a single orifice of diameter (a) $90 \mu \mathrm{m}$ and (b) $120 \mu \mathrm{m}$ as calculated from CFD. High concentration of $\mathrm{CO}_{2}$ is set on the lower part of the domain.

Replacement Image: moles_orifice.jpg

Replacement Instruction: Replace image requested

The CFD simulations also allow the determination of the Sherwood number to be used in Equation (4). In fact by comparison of Equations (4) and (14) (in this case $J_{i}^{a d v}=0$ as no convection is present):

$$
S h=\frac{\frac{\partial y_{i}}{\partial x} d_{H}}{\Delta y_{i}}=0.715
$$

Most notably this value was found to be independent both on the species selected and the hole diameter.

\subsection{Micro-perforated films characterisation}

The permeability of the membrane to nitrogen and water was estimated by means of standard correlations for polymeric materials (Sen, 2013 Robertson, 2012), since the permeability to oxygen and carbon dioxide were available. By adopting an average of the available data:

$$
\mathscr{P}_{N_{2}} \approx \frac{1}{2}\left(\frac{\mathscr{P}_{\mathrm{O}_{2}}}{3.8}+\frac{\mathscr{P}_{\mathrm{CO}_{2}}}{24}\right), \quad \mathscr{P}_{\mathrm{H}_{2} \mathrm{O}} \approx 550 \mathscr{P}_{\mathrm{N}_{2}}
$$

The tests run with an oxygen-depleted atmosphere and no cheese in the trays were used to initially check the model predictions in terms of fluxes. The comparison between the measured oxygen concentration and the one predicted by the model is reported in Fig. 3. The trend is predicted correctly, demonstrating that the proposed formulation of the fluxes across the membrane is adequate.

alt-text: Fig. 3 


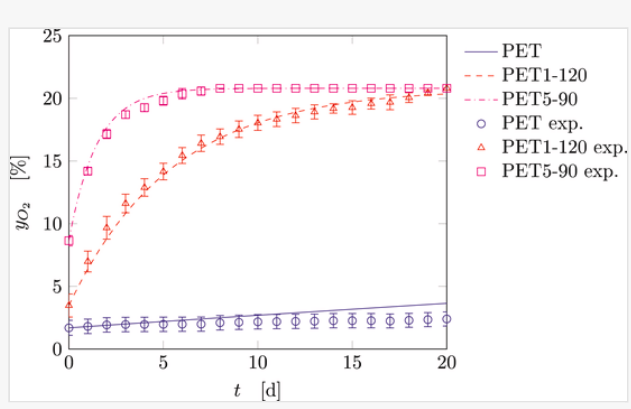

[Instruction: This figure is far from where it is first referenced to in the proof/pdf.]Comparison of the model predictions of oxygen molar fraction and experimental data for the oxygen-depleted atmospheres in empty trays.

\subsection{Determination of kinetic parameters}

All the kinetic parameters (namely, $v_{O_{2}, \max }, K_{m, O_{2}}, K_{\mu, O_{2}}, R Q, v_{\mathrm{CO}_{2}, f}, K_{1}, K_{2}$, and $\alpha$ ) can be found via fitting of the model predictions to experimental data, using the least-squares method. Respiration parameters (all except for $\alpha$ ) can be found by fitting the model prediction of gas composition in time to the experimental data. Water generally reaches saturation quite quickly, thus its molar fraction does not provide useful information for the determination of $\alpha$. On the contrary the weight loss could give useful insights on the value of such parameter. The weight loss was approximately $1 \%$ after 55 days, which could be reproduced by the model with a value of $\alpha$ equal to 0.25 .

The data collected for the packaging with no perforations were used for the parameter fitting. The kinetic parameters were found by minimisation of the objective function:

Previous Version

$\mathcal{O}=\sum\left[\left(\begin{array}{c}\exp \\ y \\ O_{2}\end{array}-y_{\mathrm{O}_{2}}\right)^{2}+\left(y_{\mathrm{CO}_{2}}^{\exp }-y_{\mathrm{CO}_{2}}\right)^{2}\right]$

Updated Version

$\mathcal{O}=\sum\left[\left(y_{\mathrm{O}_{2}}^{\exp }-y_{\mathrm{O}_{2}}\right)^{2}+\left(y_{\mathrm{CO}_{2}}^{\exp }-y_{\mathrm{CO}_{2}}\right)^{2}\right]$

where the sum is extended to all experimental data (superscript exp refers to the experimental value, while no superscript indicates the model prediction at the same time). The obtained results are reported in Table 2 . These constants are valid at the temperature of $8^{\circ} \mathrm{C}$. The predictions of the model in terms of gas composition are compared to the experimental test in Fig. 4. The computed $R^{2}$ is 0.9995 for $\mathrm{O}_{2}$ and 0.9948 for $\mathrm{CO}_{2}$.

(i) The table layout displayed in this section is not how it will appear in the final version. The representation below is solely purposed for providing corrections to the table. To preview the actual presentation of the table, please view the Proof.

[Instruction: This table is far from where it is first referenced to in the proof/pdf.]Kinetic parameters for $£$ Taleggio cheese at $8{ }^{\circ} \mathrm{C}$ found via least squares method. $v_{\mathrm{O}_{2}, \max }$ and $v_{\mathrm{CO}_{2}, \max }$ are in $\left[\mathrm{cm}^{3} \mathrm{~d}^{-1} \mathrm{~g}^{-1}\right.$ ], while all other parameters are dimensionless.

\begin{tabular}{|l|l|}
\hline Parameter & Value \\
\hline$v_{\mathrm{O}_{2}, \max }$ & 0.6015 \\
\hline$K_{m, O_{2}}$ & 0.2384 \\
\hline$K_{\mu, O_{2}}$ & 0.5348 \\
\hline$R Q$ & 0.7287 \\
\hline$v_{\mathrm{CO}_{2}, f}$ & 0.0454 \\
\hline
\end{tabular}




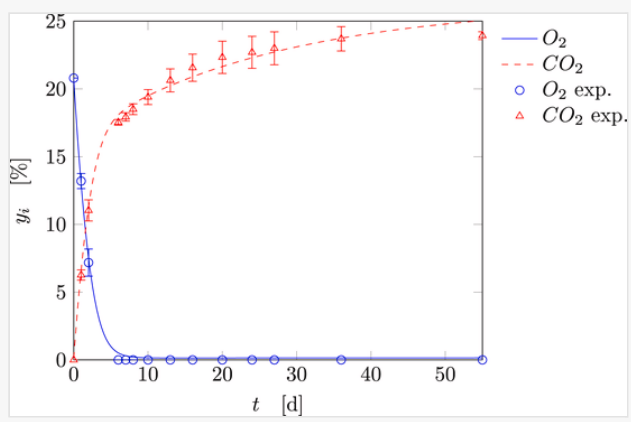

[Instruction: This figure is far from where it is first referenced to in the proof/pdf.]Comparison of the fitted model predictions of gas composition and experimental data for the non-perforated PET film.

\subsection{Validation of the model}

The experimental tests on perforated membranes were finally used to validate the proposed model both in terms of kinetics and in terms of diffusive/convective fluxes across the membrane. Fig. 5 reports the comparison of the model predictions and the experimental measurements on the gas composition inside the package over time. The agreement is satisfactory, especially for the initial part of the experiment. The computed $R^{2}$ for the three cases are reported in Table 3 for $\mathrm{O}_{2}$ and $\mathrm{CO}_{2}$. As time increases above 40 days, the experimental data deviate from the model predictions. This can be due to the change in the mould population on the crust of taleggio cheese, leading to a different anaerobic respiration kinetics.

alt-text: Fig. 5 

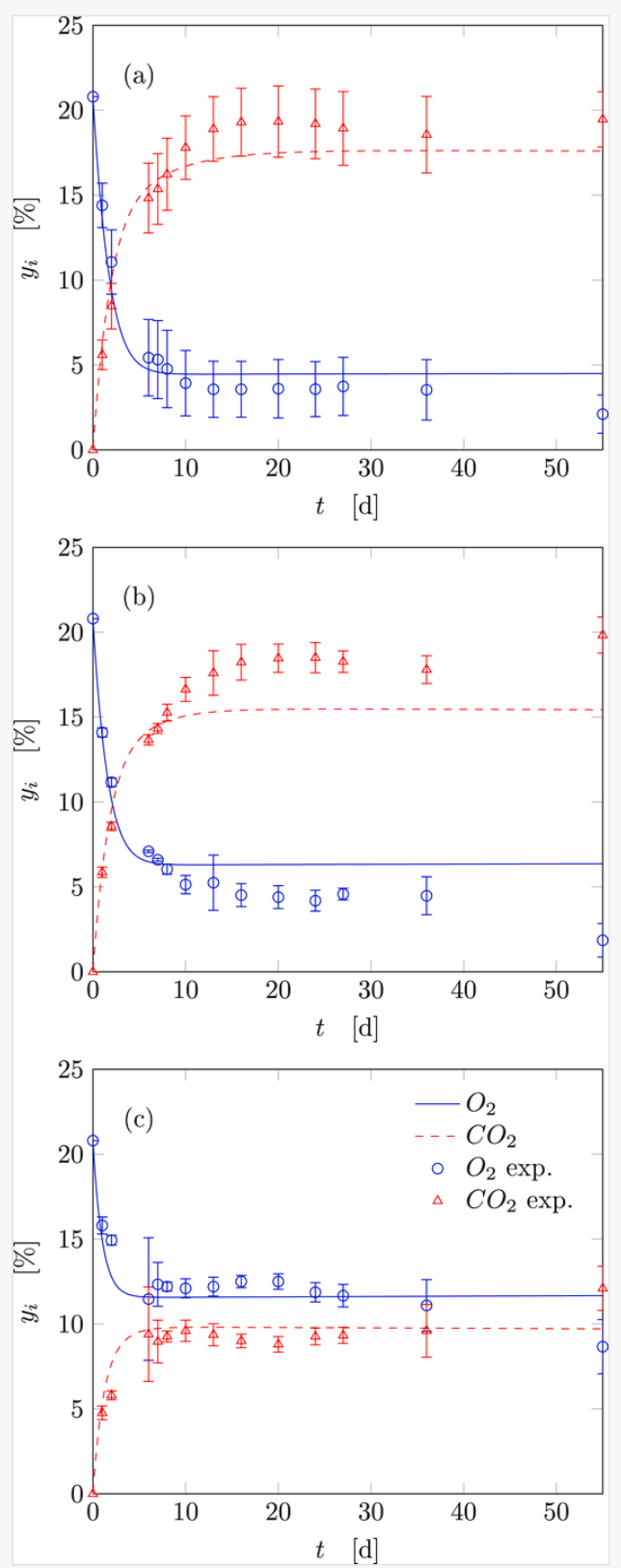

[Instruction: This figure is far from where it is first referenced to in the proof/pdf.]Comparison of the model predictions of gas composition and experimental data for the perforated packages: (a) PET1-120, (b) PET2-90, (c) PET5-90.

(i) The table layout displayed in this section is not how it will appear in the final version. The representation below is solely purposed for providing corrections to the table. To preview the actual presentation of the table, please view the Proof.

[Instruction: This table is far from where it is first referenced to in the proof $/$ pdf.] $R^{2}$ computed for the three validation cases for $\mathrm{O}_{2}$ and $\mathrm{CO}_{2}$.

\begin{tabular}{|l|l|l|}
\hline & $\boldsymbol{O}_{2}$ & $\mathrm{CO}_{2}$ \\
\hline PET1-120 & 0.9342 & 0.9436 \\
\hline PET2-90 & 0.9266 & 0.9391 \\
\hline PET5-90 & 0.8378 & 0.8999 \\
\hline
\end{tabular}

Fig. 6 reports the weight loss $(C W L)$ and the condensed water volume $\left(V_{c o n d}\right)$ over time. It should be noted that the predicted weight loss is very small, as observed experimentally, while the condensed phase volume is in the order of few millilitres, which is a good estimate of the large droplets observed hanging from the ceiling of the package. Also, the larger predicted quantity of water in PET5-90 is in agreement with the experimental formation of undesired moulds. It should be noted that the data for PET2-90 were not included for clarity purposes. Anyway, the data and model predictions are comparable to those of PET1-120. 


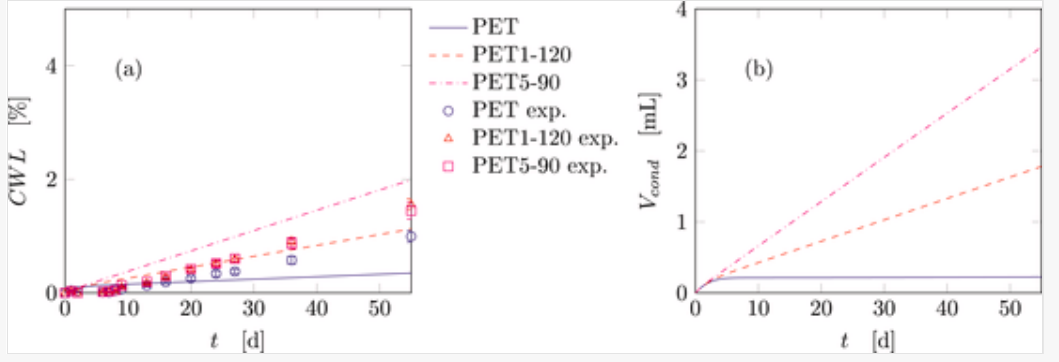

[Instruction: This figure is far from where it is first referenced to in the proof/pdf.][Instruction: This image seems at low resolution on the proof/pdf.]Model predictions for (a) the cumulative weight loss and (b) the condensate volume. Experimental data are available only for the weight loss.

From the evaluation of the fluxes for each species it is possible to find the dominant transport mechanism. For $\mathrm{CO}_{2}$ and $\mathrm{O}_{2}$ the dominant flux is the one across the holes, which is several orders of magnitude larger than the flux across the membrane thickness. The dominant mechanism for $\mathrm{H}_{2} \mathrm{O}$ is instead condensation. The fluxes $J^{M}$ and $J^{H}$ computed for water can be compared to the measured water vapour transmission ( $W V T$, details in the Supporting Information), in fact the following relationship holds true between fluxes and water vapour transmission:

$$
W V T=\frac{J_{\mathrm{H}_{2} \mathrm{O}}^{M}+J_{\mathrm{H}_{2} \mathrm{O}}^{\mathrm{H}}}{\frac{P_{\text {atm }}}{P} y_{\mathrm{H}_{2} \mathrm{O}}^{b}-y_{\mathrm{H}_{2} \mathrm{O}}} \frac{M W_{\mathrm{H}_{2} \mathrm{O}} P}{\mathscr{A}_{M} R T}
$$

which leads to a value of approximately $15.8 \mathrm{gm}^{-2} \mathrm{~d}^{-1}$ for the non-perforated membrane, $19.5 \mathrm{gm}^{-2} \mathrm{~d}^{-1}$ for PET1120, $22.1 \mathrm{gm}^{-2} \mathrm{~d}^{-1}$ for PET2-90, and $33.5 \mathrm{gm}^{-2} \mathrm{~d}^{-1}$ for PET5-90. These results are in good agreement with the measured $W V T$ from Table $\mathrm{S} 3$.

For further validation, another cheese (St. Killian) was considered, for which experimental (Rodriguez-Aguilera et al., 2011) and modelling data (Rodriguez-Aguilera et al., 2009) were available. The considered product is a soft cheese with surface mould and was sealed in a closed container and in a container with a single perforation. All geometrical parameters and conservation conditions are reported elsewhere (Rodriguez-Aguilera et al., 2011). Our model was first fitted to the case of no perforations and then used to predict the case with a perforation, as done for the 理aleggio cheese case. Fig. 7 reports the fitted gas composition (for the non-perforated packaging) and the predicted gas evolution (in a perforated packaging) against experimental data, as well as the predictions of the model proposed by RodriguezAguilera et al. (2009) based on the same cheese. Our model is able to better describe the anaerobic respiration which characterises the case of no perforations. Our proposed formulation of the respiration processes through Equations (11) and (12) allows the model to correctly predict the long-term behaviour (up to two months) of the packaging, thanks to the addition of a term which can describe anaerobic respiration. Also, the correct description of the fluxes via CFD is confirmed by the correct predictions of the perforated packaging behaviour. Therefore, the model can be deemed generalised since it can be successfully applied to different products and data sets.

alt-text: Fig. 7

Fig. 7

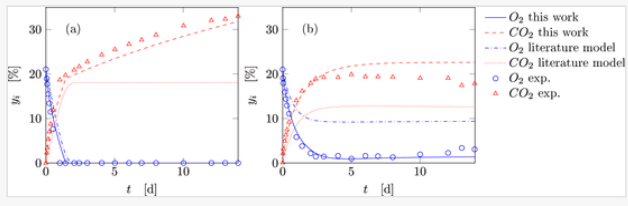

[Instruction: This figure should be paginated on two columns, as it is too small for single column.][Instruction: This figure is far from where it is first referenced to in the proof/pdf.]Application of our model for the prediction of gas evolution of packaged soft cheese (St. Killian) (Rodriguez-Aguilera et al., 2011) and comparison with a literature model (Rodriguez-Aguilera et al., 2009) in the case of (a) no perforations and (b) one perforation.

\section{Conclusions}

A packaging system based on the combination of micro-perforations and EMAP was modelled and studied to control the atmosphere inside a package for a smear short-ripened soft cheese (£Taleggio). Faleggie This cheese possess a 
complex surface microbiota which contribute to its typical sensorial characteristic and shows different respiration

kinetics as function of the gas concentration $\left(\mathrm{O}_{2}\right.$ and $\left.\mathrm{CO}_{2}\right)$.

Specifically, the developed mathematical model is able to describe and predict the headspace atmosphere evolution under isothermal conditions. Fluxes through the micro-perforations were estimated using CFD. Considering the generalised nature of the models, it can be exploited to identify the optimal perforation configuration for any type of micro-perforated packaging systems (e.g. stiff systems like boxes or flexible ones like bags) and for any foods.

The efficiency of the model was experimentally validated by measuring the gas evolution in four different $\underline{\text { Taleggio }}$ packaging configurations, one without and three with micro-perforations. Taking into account both aerobic and anaerobic respiration processes, the proposed model is able to efficiently predict the gas concentration for the whole product shelf-life (up to 55 days).

The obtained experimental results showed that it is possible to modulate gas permeability values through microperforations in such a way to achieve the desired equilibrium internal gas composition, and to get an optimal condition for extended shelf-life.

Overall, the micro-perforation technology and an accurate prediction of the headspace atmosphere evolution by a mathematical model become a very useful and powerful tool for the quick development of an ad hoc packaging solutions for delicate and valuable food products, paving the road to potential new applications in other food matrices.

\section{Declaration of competing interest}

The authors declare that they have no known competing financial interests or personal relationships that could have appeared to influence the work reported in this paper.

\section{Appendix A Supplementary data}

Supplementary data to this article can be found online at https://doi.org/10.1016/j.jpoodeng.2021.110765.

\section{Authore statement}

Conceptualization Luigi De Nardo, Methodology Luigi De Nardo, Federico Florit, Renato Rota Formal analysis Federico Florit, Renato Rota Investigation Andrea Fiorati, Filippo Ghisoni, Gabriele Pozzoli, Data curation Andrea Fiorati, Filippo Ghisoni, Gabriele Pozzoli Writing - Original Draft Federico Florit, Andrea Fiorati, Filippo Ghisoni, Gabriele Pozzoli Writing - Review \& Editing Renato Rota, Luigi De Nardo Visualization Federico Florit, Andrea Fiorati, Supervision Luigi De Nardo.

\section{References[Instruction: hyperlink errors are present in references without DOI.]}

The corrections made in this section will be reviewed and approved by a journal production editor. The newly added/removed references and its citations will be reordered and rearranged by the production team.

Bossi, E., Tana, F., Punta, C., Cigada, A., Nardo, L.D., 2016. Flexible hybrid coatings with efficient antioxidation properties. Food Packaging and Shelf Life 10, 106-114. doi:10.1016/j.fpsl.2016.10.002.

Castellanos, D.A., Cerisuelo, J.P., Hernandez-Muñoz, P., Herrera, A.O., Gavara, R., 2016a. Modelling the evolution of $\mathrm{O} 2$ and $\mathrm{CO} 2$ concentrations in MAP of a fresh product: application to[Instruction: Doi is missing: 10.1016/j.jfoodeng.2015.07.019] tomato. J. Food Eng. 168, 84-95.

Castellanos, D.A., Herrera, D.R., Herrera, A.O., 2016b. Modelling water vapour transport, transpiration and weight loss in a perforated modified atmosphere packaging for feijoa fruits. Biosyst. Eng. 151, 218-230. doi:10.1016/j.biosystemseng.2016.08.015.

Castellanos, D.A., Mendoza, R., Gavara, R., Herrera, A.O., 2017. Respiration and ethylene generation modeling of "hass" avocado and feijoa fruits and application in modified atmosphere packaging. Int. J. Food Prop. 20 (2), 333-349. doi:10.1080/10942912.2016.1160921.

Ciccullo, F., Cagliano, R., Bartezzaghi, G., Perego, A., 2021. Implementing the circular economy paradigm in the agri-food supply chain: the role of food waste prevention technologies. Resour. Conserv. Recycl. 164, 105114. doi:10.1016/j.resconrec.2020.105114. 
Dermiki, M., Ntzimani, A., Badeka, A., Savvaidis, I.N., Kontominas, M.G., 2008. Shelf-life extension and quality attributes of the whey cheese "myzithra kalathaki" using modified atmosphere packaging. LWT-Food Science and Technology 41 (2), 284-294. doi:10.1016/j.lwt.2007.02.014.

Esmer, O.K., Balkir, P., Seckin, A.K., Irkin, R., 2009. The effect of modified atmosphere and vacuum packaging on the physicochemical, microbiological, sensory and textural properties of crottin de chavignol cheese. Food Sci. Technol. Res. 15 (4), 367-376. doi:10.3136/fstr.15.367.

Fontana, C., Cappa, F., Rebecchi, A., Cocconcelli, P.S., 2010. Surface microbiota analysis of taleggio, gorgonzola, casera, scimudin and formaggio di fossa Italian cheeses. Int. J. Food Microbiol. 138 (3), 205-211. doi:10.1016/j.ijfoodmicro.2010.01.017.

Geysen, S., Escalona, V.H., Verlinden, B.E., Nicolaï, B.M., 2007. Modelling the effect of super-atmospheric oxygen and carbon dioxide concentrations on the respiration of fresh-cut butterhead lettuce. J. Sci. Food Agric. 87 (2), 218-226. doi:10.1002/jsfa.2696.

Ghosh, T., Dash, K., 2020. Modeling on respiration kinetics and modified atmospheric packaging of fig fruit. Food Measure 14, 1092-1104. doi:10.1007/s11694-019-00359-2.

Gobbetti, M., Lowney, S., Smacchi, E., Battistotti, B., Damiani, P., Fox, P., 1997. Microbiology and biochemistry of taleggio cheese during ripening. Int. Dairy J. 7 (8), 509-517. doi:10.1016/S09586946(97)00044-7.

Hollingshead, C., Johnson, M., Barfuss, S., Spall, R., 2011. Discharge coefficient performance of venturi, standard concentric orifice plate, v-cone and wedge flow meters at low Reynolds numbers. J. Petrol. Sci. Eng. 78 (3), 559-566. doi:10.1016/j.petrol.2011.08.008.

Hussein, Z., Caleb, O.J., Opara, U.L., 2015. Perforation-mediated modified atmosphere packaging of fresh and minimally processed produce - a review. Food Packaging and Shelf Life 6, 7-20. doi:10.1016/j.fpsl.2015.08.003.

Khoshgozaran, S., Azizi, M., Bagheripoor-Fallah, N., 2012. Evaluating the effect of modified atmosphere packaging on cheese characteristics: a review. Dairy Sci. Technol. 92, 1-24. doi:10.1007/s13594-011-0044-3.

Marcos, B., Bueno-Ferrer, C., Fernández, A., 2016. Innovations in packaging of fermented food products. In: Ojha, K.S., Tiwari, B.K. (Eds.), Novel Food Fermentation Technologies. Springer[Instruction: Doi is missing: 10.1007/978-3-319-42457-6_15] International Publishing, pp. 311-333.

Panton, R.L., 2013. Incompressible Flow. fourth ed. John Wiley \& Sons, Ltd. doi:10.1002/9781118713075.

Papaioannou, G., Chouliara, I., Karatapanis, A.E., Kontominas, M.G., Savvaidis, I.N., 2007. Shelf-life of a Greek whey cheese under modified atmosphere packaging. Int. Dairy J. 17 (4), 358-364. doi:10.1016/j. idairyj.2006.04.001.

Rennie, T., Tavoularis, S., 2009a. Perforation-mediated modified atmosphere packaging: Part i. development of a mathematical model. Postharvest Biol. Technol. 51 (1), 1-9. doi:10.1016/j.postharvbio.2008.06.007.

Rennie, T., Tavoularis, S., 2009b. Perforation-mediated modified atmosphere packaging. [Instruction: "Part" should be capitalized.]part ii. implementation and numerical solution of a mathematical model. Postharvest Biol. Technol. 51 (1), 10-20. doi:10.1016/j.postharvbio.2008.06.012.

SonRobertson, G.L.R.G.L., 20132012. Food Packaging Principles and Practice. Third Edition [Instruction: doi is missing:

10.1201/b21347](3rd ed.)_Taylor \& Francis Group.

Rodriguez-Aguilera, R., Oliveira, J.C., 2009. Review of design engineering methods and applications of active and modified atmosphere packaging systems. Food Engineering Reviews 1 (1), 66-83. doi:10.1007/s12393-009-9001-9.

Rodriguez-Aguilera, R., Oliveira, J.C., Montanez, J.C., Mahajan, P.V., 2009. Gas exchange dynamics in modified atmosphere packaging of soft cheese. J. Food Eng. 95 (3), 438-445. doi:10.1016/j.jfoodeng.2009.06.021. 
Rodriguez-Aguilera, R., Oliveira, J.C., Montanez, J.C., Mahajan, P.V., 2011. Effect of modified atmosphere packaging on quality factors and shelf-life of surface mould ripened cheese: Part i constant temperature. LWT - Food Sci. Technol. (Lebensmittel-Wissenschaft -Technol.) 44 (1), 330-336. doi:10.1016/j.lwt.2010.06.015.

Ruggeri, E., Kim, D., Cao, Y., Farè, S., De Nardo, L., Marelli, B., 2020. A multilayered edible coating to extend produce shelf life. ACS Sustain. Chem. Eng. 8 (38), 14312-14321. doi:10.1021/acssuschemeng.0c03365.

Song, Y., Vorsa, N., Yam, K.L., 2002. Modeling respiration-transpiration in a modified atmosphere packaging system containing blueberry. J. Food Eng. 53 (2), 103-109. doi:10.1016/S0260-8774(01)00146-7.

Sousa-Gallagher, M., Mahajan, P., Mezdad, T., 2013. Engineering packaging design accounting for transpiration rate: model development and validation with strawberries. J. Food Eng. 119 (2), 370-376. doi:10.1016/j.jf foodeng.2013.05.041.

E. Tirloni, S. Stella, C. Bernardi, D. Mazzantini, F. Celandroni, E. Ghelardi, Identifieation and pathogenie potential of baeillus cereus strains isolated from a dairy processing plant producing pdo taleggio cheese, Mieroorganisms 8 (6). doi:10.3390/mierganism8060949-Tirloni,_E.,_Stella, S.,_Bernardi,_C.,_Mazzantini,_D.,_Celandroni,_F., Ghelardi,_E.,2020. Identification and pathogenic potential of bacillus cereus strains isolated from a dairy. processing plant producing.pdo taleggio cheese. Microorganisms 8 (6), 949. doi:10.3390/microorganisms8060949.

Winotapun, C., Kerddonfag, N., Kumsang, P., Hararak, B., Chonhenchob, V., Yamwong, T., Chinsirikul, W., 2015. Microperforation of three common plastic films by laser and their enhanced oxygen transmission for fresh produce packaging. Packag. Technol. Sci. 28 (4), 367-383. doi:10.1002/pts.2108.

Xanthopoulos, G., Koronaki, E., Boudouvis, A., 2012. Mass transport analysis in perforation-mediated modified atmosphere packaging of strawberries. J. Food Eng. 111 (2), 326-335. doi:10.1016/j.jfoodeng.2012.02.016.

Youssef, A.M., Assem, F., El-Sayed, S., Salama, H., Abd El-Salam, M., 2017. Utilization of edible films and coatings as packaging materials for preservation of cheeses. Journal of Packaging Technology and Research 1 (2), 87-99. doi:10.1007/s41783-017-0012-3.

Zeuthen, P., 2002. 8 - safety criteria for minimally processed foods. In: Ohlsson, T., Bengtsson, N. (Eds.), Minimal Processing Technologies in the Food Industries, Woodhead Publishing Series in Food Science, Technology and Nutrition. Woodhead Publishing, pp. 196-218. doi:10.1533/9781855736795.196.

\section{Highlights}

- A generalised model for equilibrium modified atmosphere packaging (EMAP) with micro-perforation is developed.

- Computational fluid dynamics (CFD) is used to predict diffusive fluxes through micro-perforations.

- Taleggio cheese EMAP experiments are presented.

- The model correctly describes the gas behavior inside the packaging for taleggio cheese and other soft cheese.

\section{Appendix A Supplementary data}

The following is the Supplementary data to this article: 


\section{Queries and Answers}

\section{Q1}

Query: Correctly acknowledging the primary funders and grant IDs of your research is important to ensure compliance with funder policies. Please make sure that funders are mentioned accordingly.

Answer: Reviewed

\section{Q2}

Query: Please confirm that the provided email "luigi.denardo@polimi.it" is the correct address for official communication, else provide an alternate e-mail address to replace the existing one, because private e-mail addresses should not be used in articles as the address for communication.

Answer: Reviewed

Q3

Query: Tables were not sequentially cited in the text, and have been renumbered both in the text and in the artwork. Please check, and correct if necessary.

Answer: The tables were checked and the numbering is correct.

\section{Q4}

Query: Please confirm that given names and surnames have been identified correctly and are presented in the desired order and please carefully verify the spelling of all authors' names.

Answer: Reviewed

\section{Q5}

Query: Your article is registered as a regular item and is being processed for inclusion in a regular issue of the journal. If this is NOT correct and your article belongs to a Special Issue/Collection please contact b.sims@elsevier.com immediately prior to returning your corrections.

Answer: Yes 